\title{
FERMILAB-TM-2228
}

\section{Time-of-flight measurement in the DZero Central Fiber Tracker}

\author{
J. Estrada, C. García, B. Hoeneisen and P. Rubinov
}

$\mathrm{D} \emptyset$ note 4202 ,

20 July 2003

\begin{abstract}
We continue evaluation of the new electronics developed for the Central Fiber Tracker and Preshower detectors. With the custom TriP chip and MCM II we have measured the position of the hits along the fiber by comparing the time of arraival of the photons at the VLPC with the expected timming relative to the beam. The measured rms resolution at the center of the fibers is $46 \mathrm{~cm}$ for hits with more than 8 photo-electrons and is dominated by the statistics of photon arrival time. The corresponding resolution near the ends of the fibers (where more photoelectrons are collected) is calculated to be of order $27 \mathrm{~cm}$. With a second submission of the TriP chip to add the time-of-flight measuring capability we will effectively double the number of channels in the central fiber tracker. This capability will increase the maximum luminosity at which D0 can do tracking from $\approx 100 \cdot 10^{30} \mathrm{~cm}^{-2} \mathrm{~s}^{-1}$ to $\approx 200 \cdot 10^{30} \mathrm{~cm}^{-2} \mathrm{~s}^{-1}$ ) (at a bench mark tracking specification). The cost of replacing the electronics is of order $\$ 500 \mathrm{~K}$ and the necessary lead time is 1.5 years.
\end{abstract}




\section{Introduction}

The original plan of operation of the Tevatron was to switch from 396ns bunch crossing in Run IIa to 132ns bunch crossing in Run IIb. The "SIFT" chips in the Multi Channel Modules (MCM's) in the Analog Front End boards (AFE's) of the Central Fiber Tracker (CFT) and Preshower detectors can not operate at 132ns bunch crossing. [1] For this reason a new MCM with a new custom "TriP" chip was designed[2], built and tested.[3, 4] The first (and only) submission of the chip and the MCM met specifications and 7000 chips are in-hand, enough to populate all new AFE's. However the current plan of the Tevatron is to abandon operation at 132ns bunch crossing. At 396ns bunch crossing the analog pipeline of the TriP chip is sufficiently deep to store the time at which the discriminators fire in addition to the amplitude of the pulses. This feature permits a coarse measurement of the $z$-coordinate in the CFT after the L1 trigger accept. This $z$-coordinate measurement reduces the combinatoric background of tracking, speeds up the tracking algorithm, and allows the CFT to operate at higher luminosity. The added capability of the TriP chip requires a new submission of the chip.[3]

In this note we describe time-of-flight measurements in the CFT using MCM IIb[3, 4] with two TriP chips.

\section{Spectra, noise and gain}

We used a test stand on the 3rd floor of the $\mathrm{D} \emptyset$ Assembly Building. This test stand has a computer, a Stand-Alone-Sequencer, an AFE I on a Visible Photon Light Counter (VLPC) cryostat and casette, and a CFT cylinder of $30 \mathrm{~cm}$ radius and $250 \mathrm{~cm}$ length. The light guide connecting the scintillating fibers to the casette was $11 \mathrm{~m}$ long. A histogram of the amplitude of the output of one channel of a TriP chip with a LED light source is shown in Figure 1. From the fit we obtain a gain of 21 ADC counts per photo-electron. From a fit to the pedestal corresponding to a VLPC that is not illuminated we obtain an rms noise of 2.7 ADC counts. The charge per ADC count is 0.486fC.[4] Therefore we obtain an electronic plus VLPC rms noise of $1.17 \mathrm{fC}$, and a VLPC gain of $21 \cdot 0.486 /\left(1.602 \cdot 10^{-4}\right)=63700$ at a bias voltage of $7.2 \mathrm{~V} .{ }^{1}$

\footnotetext{
${ }^{1}$ This is the gain of the VLPC including the $\approx 35 \mathrm{pF}$ stray capacitance of the flex cable and the $100 \mathrm{pF}$ coupling capacitor. The corresponding gain at $7.0 \mathrm{~V}$ is $63700 / 1.127=56500$. (This "composite" gain was quoted in [5].) The gain of the VLPC on its own is 56500 . $(100+35) / 100=76000$ at $7.0 \mathrm{~V}$.
} 


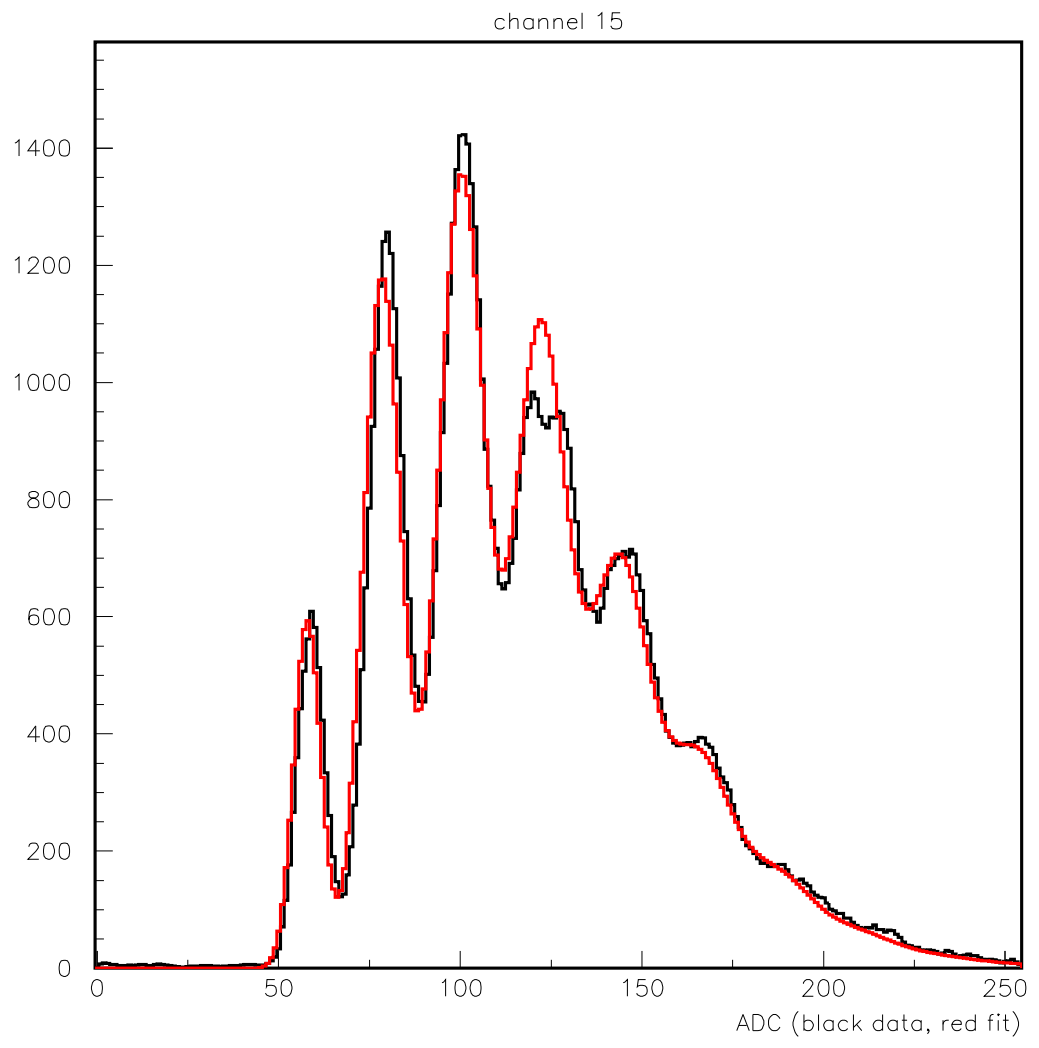

Figure 1: Histogram of ADC output of one channel of a TriP chip taken with a LED light source and fit. The peaks correspond to 0 (i.e. pedestal), 1, 2, $3,4,5$ and 6 photo-electrons. The bias voltage on the VLPC is $7.2 \mathrm{~V}$. The VLPC gain (including flex cable capacitance) at this bias voltage is 63700 . The temperature is $9.0 \mathrm{~K}$. The registers of the Trip chip where set to the values listed in Table 1. 


\begin{tabular}{|c|c|c|c|c|c|c|c|c|c|c|c|}
\hline R1 & R2 & R3 & R4 & R5 & R6 & R7 & R8 & R9 & R10 & R11 & R12 \\
\hline 130 & 120 & 20 & 160 & 138 & 24 & 42 & 10 & 160 & 215 & 454 & 454 \\
\hline
\end{tabular}

Table 1: Registers in TriP chips. See [3] for a description of the registers.

\section{Time-of-flight measurements}

We used cosmic muons triggered with two counters in coincidence. We used the FPGA implementation for MCM IIb with 132ns cycle with one discriminator bank of the time-multiplexed pair always enabled and the discriminator outputs of 16 channels of TriP 0 and 16 channels of TriP 1 ORed to increase the rate of data acquisition. The original file of the FPGA implementation is FPGAMCMIIB_9_MAY_2001.ZIP, and the modified file is MCMIIB_Z_11JUL2003.ZIP (which are available in D0server4). The VLPC bias voltage is $7.0 \mathrm{~V}$ and the TriP chip registers are set as shown in Table 1. The discriminator threshold is set at 1.5 photo-electrons. The scatter plot of amplitude (ADC counts) vs time of discriminator firing is shown in Figure 2. In Figure 3 we show a histogram of the time at which the discriminators fire (with respect to the coincidence obtained by the cosmic muon detectors) for hits with an amplitude greater than 200 ADC counts corresponding to 8 photo-electrons. Standard deviations are shown in Figure 4. For 8 or more photo-electrons the standard deviation is $2.8 \mathrm{~ns}$ corresponding to a $z$-coordinate rms resolution of $46 \mathrm{~cm}$ and scales with the number $n$ of photo-electrons as $\propto \sqrt{n}$. The velocity of light in the fibers is $16.6 \mathrm{~cm} / \mathrm{ns}$. The time jitter attributable to the trigger counters was negligible, see Figure 5. In the following Section we shall see that the electronic noise also contributes negligibly. The resolution of the time-of-flight measurement is due to the random arrival time of photons (see Table 9 of [3]). This measurement was done at the center of the scitillator fibers. At the near and far ends, where more light is collected due to oblique incidence of tracks, we expect an $\mathrm{rms}$ resolution of $\approx 27 \mathrm{~cm}$ (see Table 9 of [3]).

A histogram of the amplitude of pulses is shown in Figure 6. This histogram confirms that the discriminator threshold is set at 1.5 photo-electrons.

\section{Electronic noise}

We pulsed one channel of the TriP chip with $60 \mathrm{fC}$ corresponding to 7 photoelectrons (at the VLPC bias of $7.0 \mathrm{~V}$ ). The pedestal is at 60 ADC counts. We used cosmic muons to trigger the charge injection so that it arrived at 


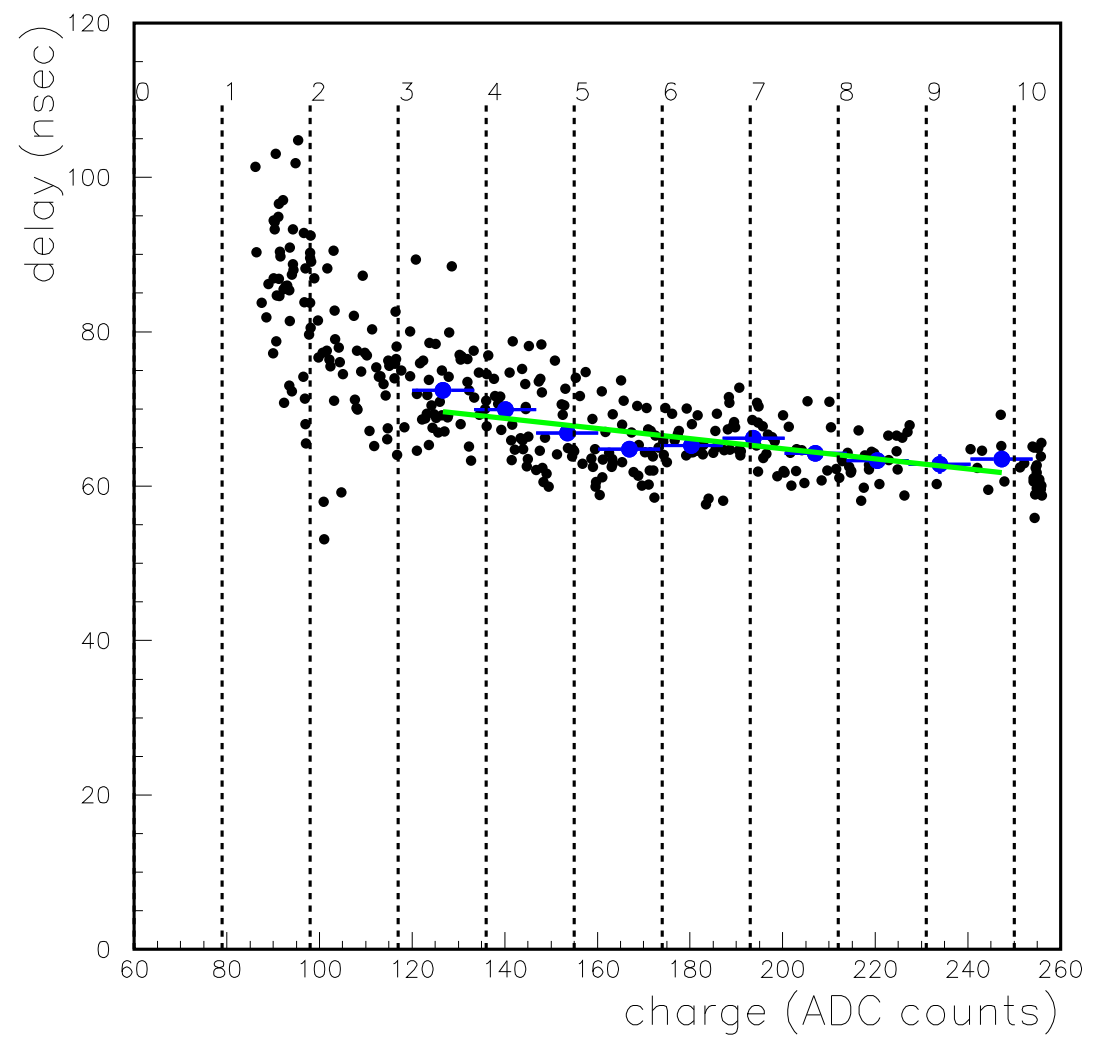

Figure 2: Scatter plot of amplitude (ADC counts) vs time of discriminator firing for muons within a 30ns acquisition window. The vertical lines indicate the number of photo-electrons. Sum of 16 channels of TriP 0 and 16 channels of TriP 1. The solid line indicates the discriminator time-walk to be corrected off-line. 


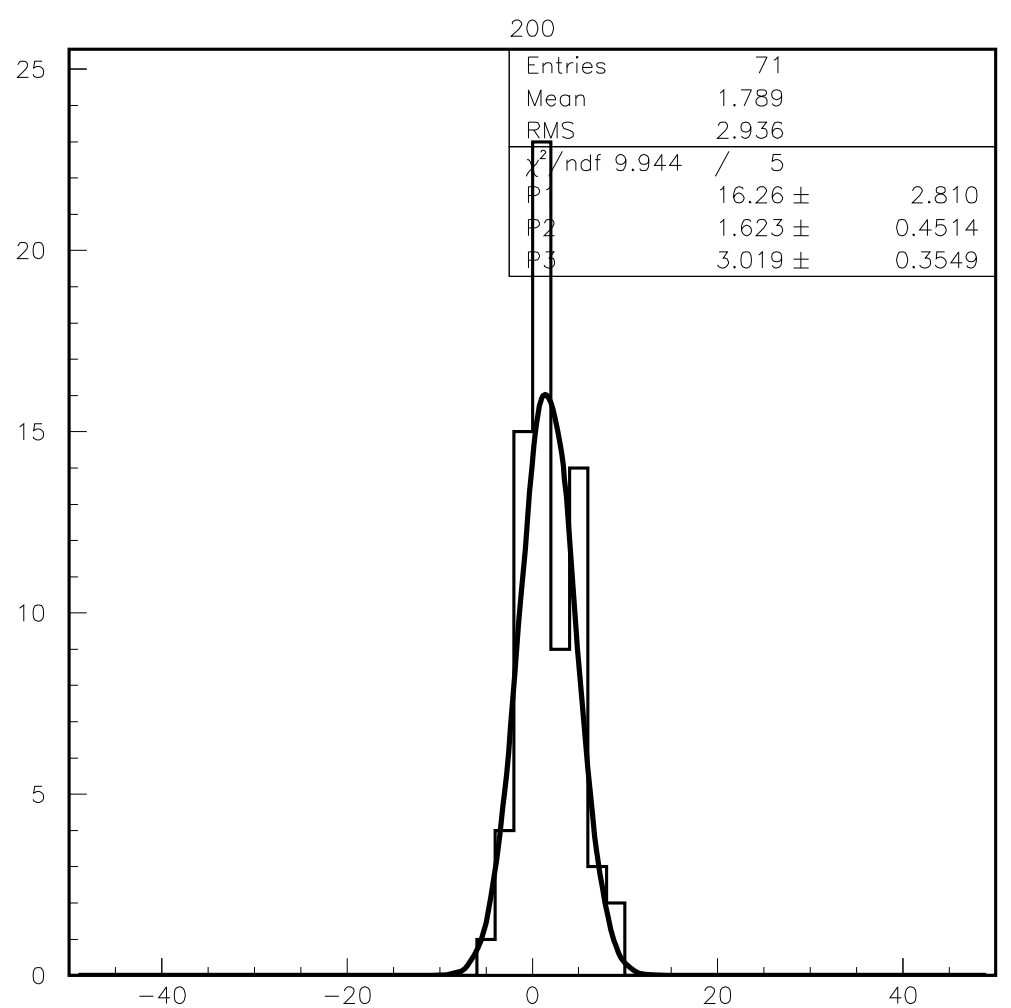

Figure 3: Histogram of the time of discriminator firing for hits with more than 200 ADC counts ( 8 photo-electrons). The rms is $2.94 \mathrm{~ns}$ and the standard deviation of the fit is $3.02 \mathrm{~ns}$. 

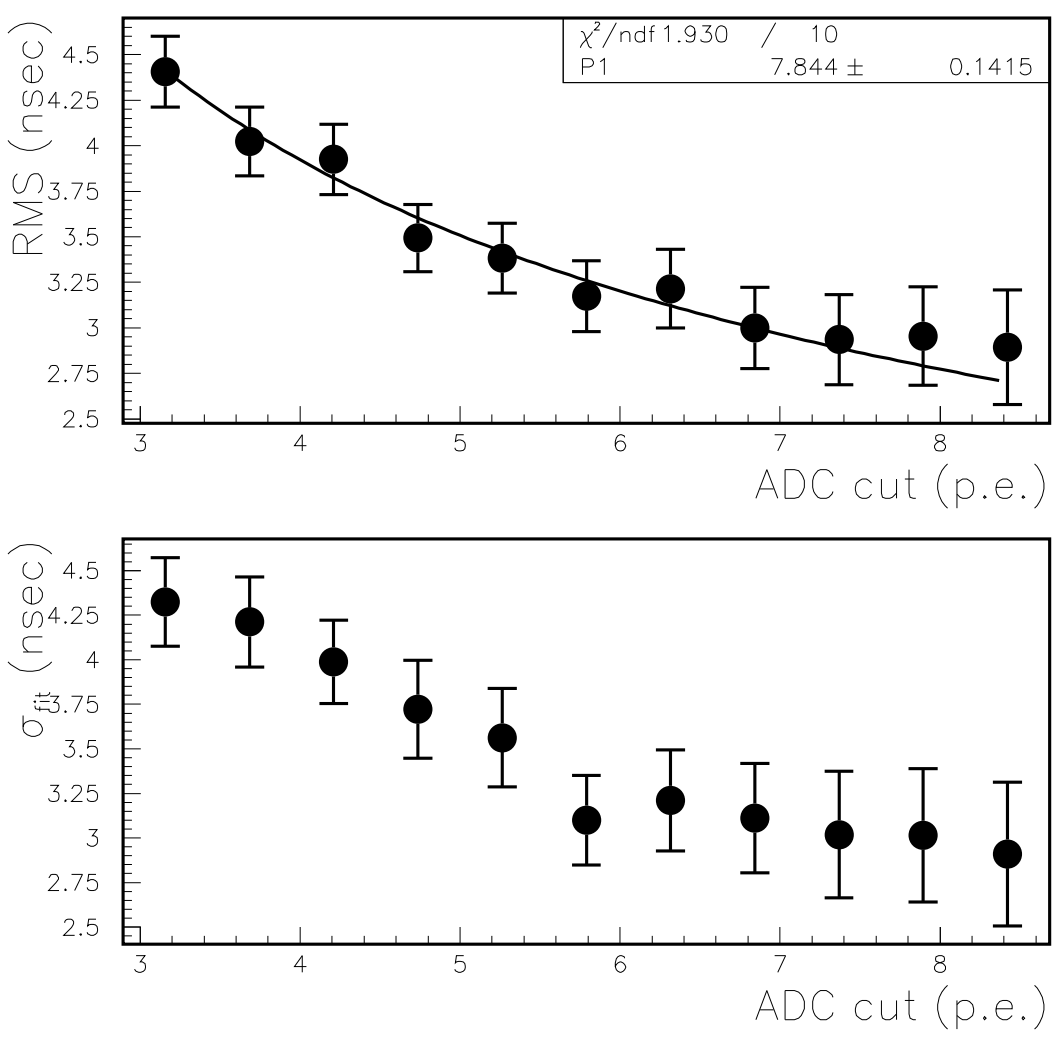

Figure 4: Standard deviation of switching times as a function of the ADC cut expressed in photo-electrons. Data (upper plot) and Gaussian fit of data (lower plot). 


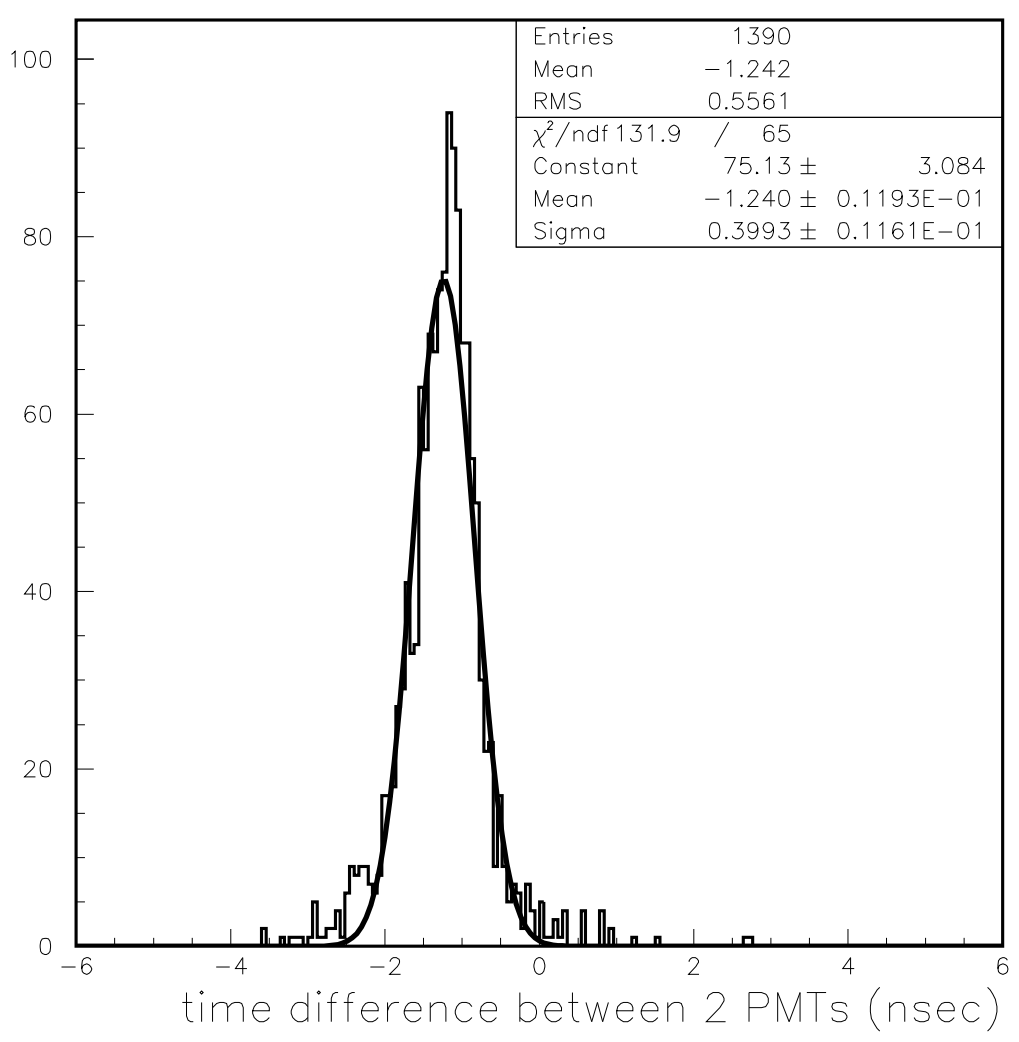

Figure 5: Cosmic muons were detected by two scintillators with a Philips XP2282 photomultiplier which were required to be in coincidence with an amplitude greater than $20 \mathrm{mV}$. Shown is a histogram of the time difference of the two photomultiplier signals at $10 \mathrm{mV}$. The rms time jitter of the muon trigger is $0.56 \mathrm{~ns}$. 


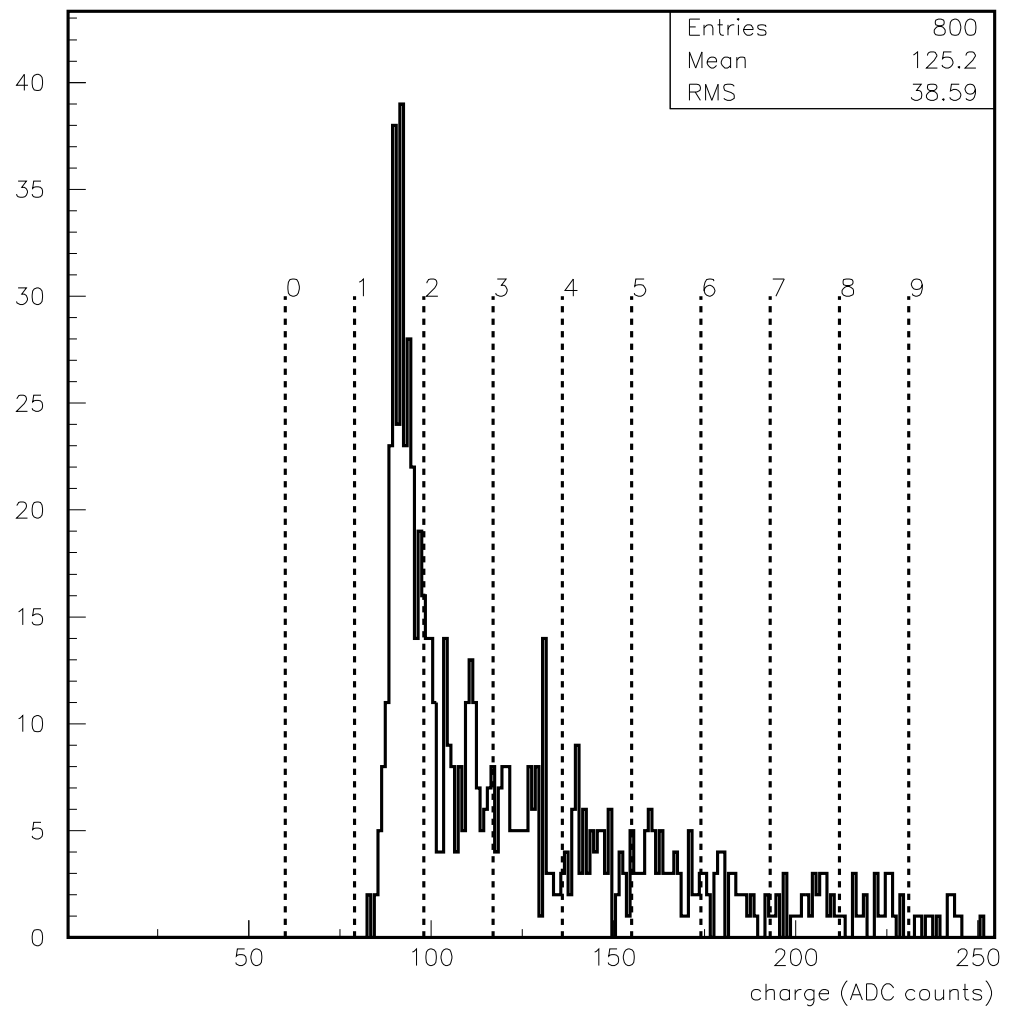

Figure 6: Histogram of amplitude in ADC counts. The vertical lines indicate the number of photoelectrons. 
random times with respect to the TriP chip clocks. The results are shown in Figures 7 and 8 . The rms noise of the discriminators was 0.42 ns over a useful acquisition window of 40ns.

Repeating the measurement with an injection of $30 \mathrm{fC}$ corresponding to 3.5 photo-electrons we obtained an rms noise of $0.66 \mathrm{~ns}$ as shown in Figure 9. The mean of the histogram in Figure 9 occurs $3.5 \mathrm{~ns}$ before the mean in Figure 8. This discriminator time-walk is as expected.[3]

In conclusion, the electronic noise of the VLPC and TriP chip is negligible compared to the fluctuation in arrival time of the photons.

\section{$5 \quad$ Is the time-of-flight measurement worth while?}

In Figure 10 and Table 2 we show the measured mean occupancy of the inner layer of the fiber tracker as a function of luminosity and ADC cut.[7] Note in Figure 10 that the relation between luminosity and occupancy is linear in the measured range. In Table 3 we show the luminosity plan of the Tevatron[8] and the corresponding occupancies of the inner layer of the CFT obtained by extrapolating linearly the data in Table 2 .

The number of fake tracks found in the stand-alone CFT is of order[6]

$$
n_{\text {fake }} \approx 6 \cdot 10^{10} \cdot \epsilon^{\left(n_{A}+n_{S}\right)} \cdot \frac{B_{\text {mag }}}{p_{\text {Tmin }}}
$$

where $\epsilon$ is the fiber occupancy, i.e. the fraction of fibers with hits, $B_{\text {mag }}$ is the magnetic field in Tesla, $p_{\text {Tmin }}$ is the minimum transverse momentum of the searched tracks in $\mathrm{GeV} / \mathrm{c}$, and $n_{A}\left(n_{S}\right)$ is the number of axial (stereo) doublet hits required on track. Note that for this back-of-the-envelope calculation we take the same occupancy $\epsilon$ for all layers of the CFT and set it equal to the mean occupancy of the inner layer as given in Table 3. This is done for simplicity and to somehow take into account particularly busy (and interesting) events.

As a benchmark let us take $B_{\text {mag }}=2 \mathrm{~T}, p_{\text {Tmin }}=0.5 \mathrm{GeV} / \mathrm{c}, n_{A}=n_{S}=8$ and $n_{\text {fake }}=1$. Then the maximum occupancy at which the CFT can do stand-alone tracking is $\epsilon \approx 0.19$.

If we include the Silicon Microstrip Tracker (SMT) as part of the tracking (not only as a vertex detector) the right hand side of Equation (1) becomes multiplied by a factor of order

$$
\times 160 \epsilon_{S i}^{n_{S i}}
$$

where $\epsilon_{S i}$ is the SMT occupancy and $n_{S i}$ is the number of hits in the SMT. Extrapolating the CFT occupancy into the SMT we would guess $\epsilon_{S i} \approx \epsilon / 50 \approx$ 

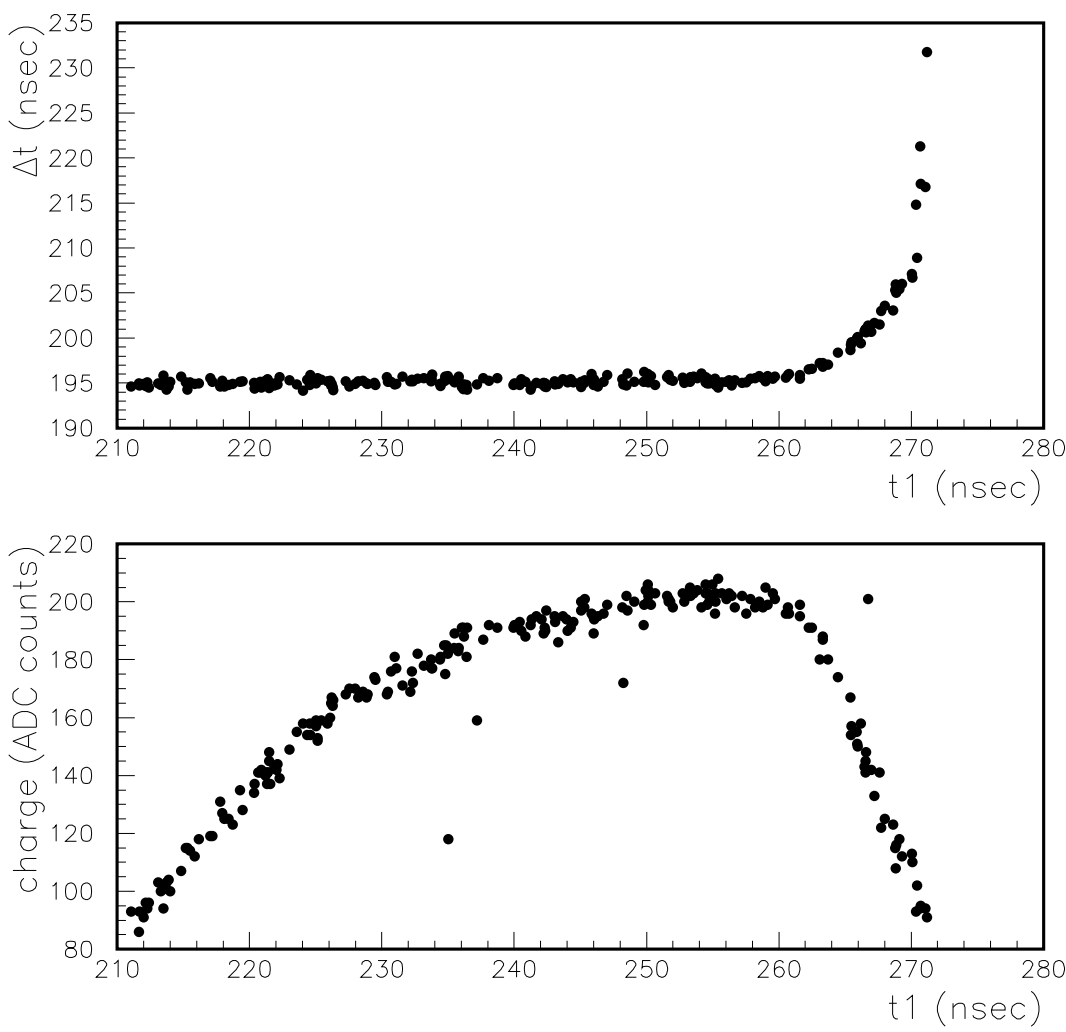

Figure 7: Injection of $60 \mathrm{fC}$ into one TriP chip channel. Time of injection relative to TriP chip clocks (zero is arbitrary and sign is reversed) vs time of discriminator firing (upper plot) and amplitude in ADC counts (lower plot). The tail with time $<-260 \mathrm{~ns}$ corresponds to injection before the acquisition window. Late injection (time $>-230 \mathrm{~ns}$ ) results in incomplete integration and a drop in amplitude. 

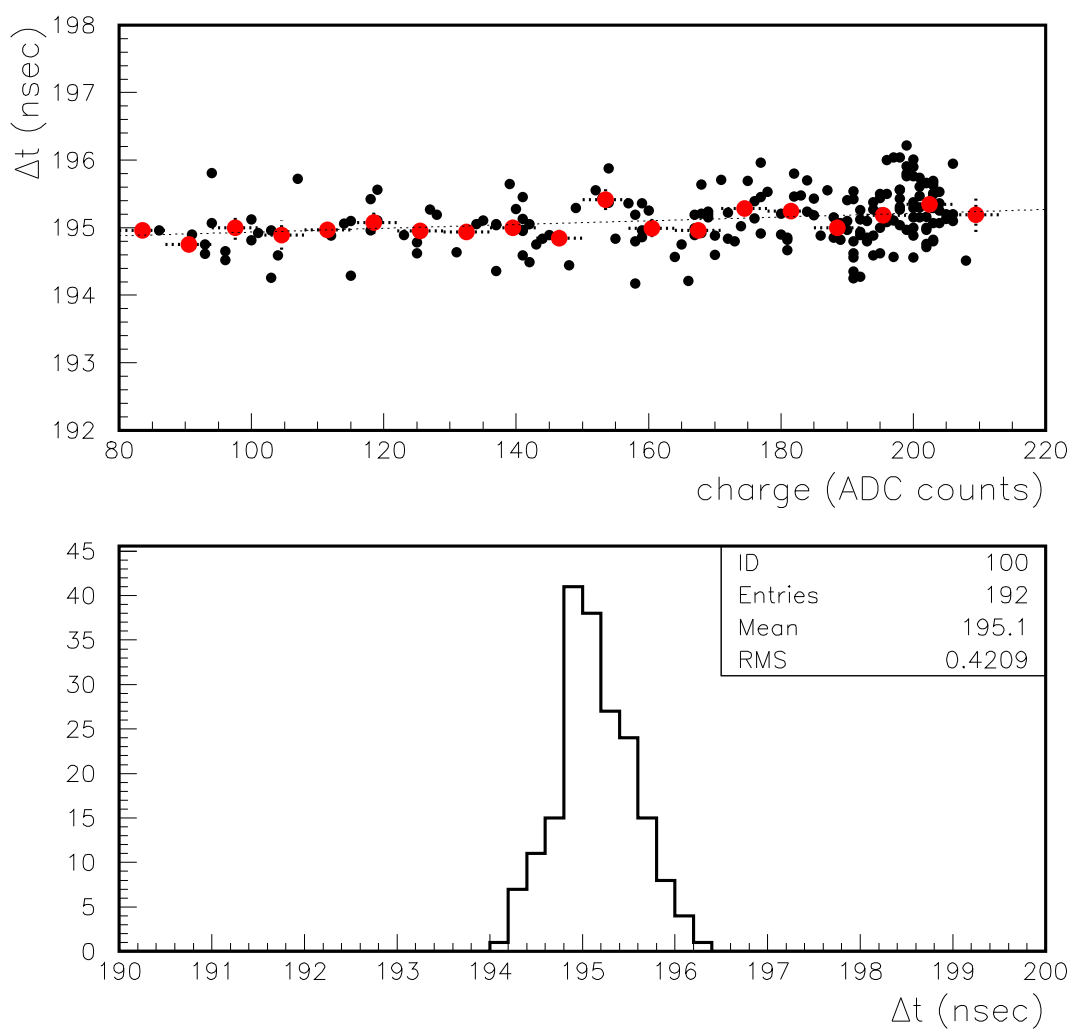

Figure 8: Upper plot: Time of discriminator firing as a function of ADC counts. The corresponding histogram of time (lower plot) has an rms noise of $0.42 \mathrm{~ns}$. The injected charge is $60 \mathrm{fC}$ at random times within a $30 \mathrm{~ns}$ acquisition window. 

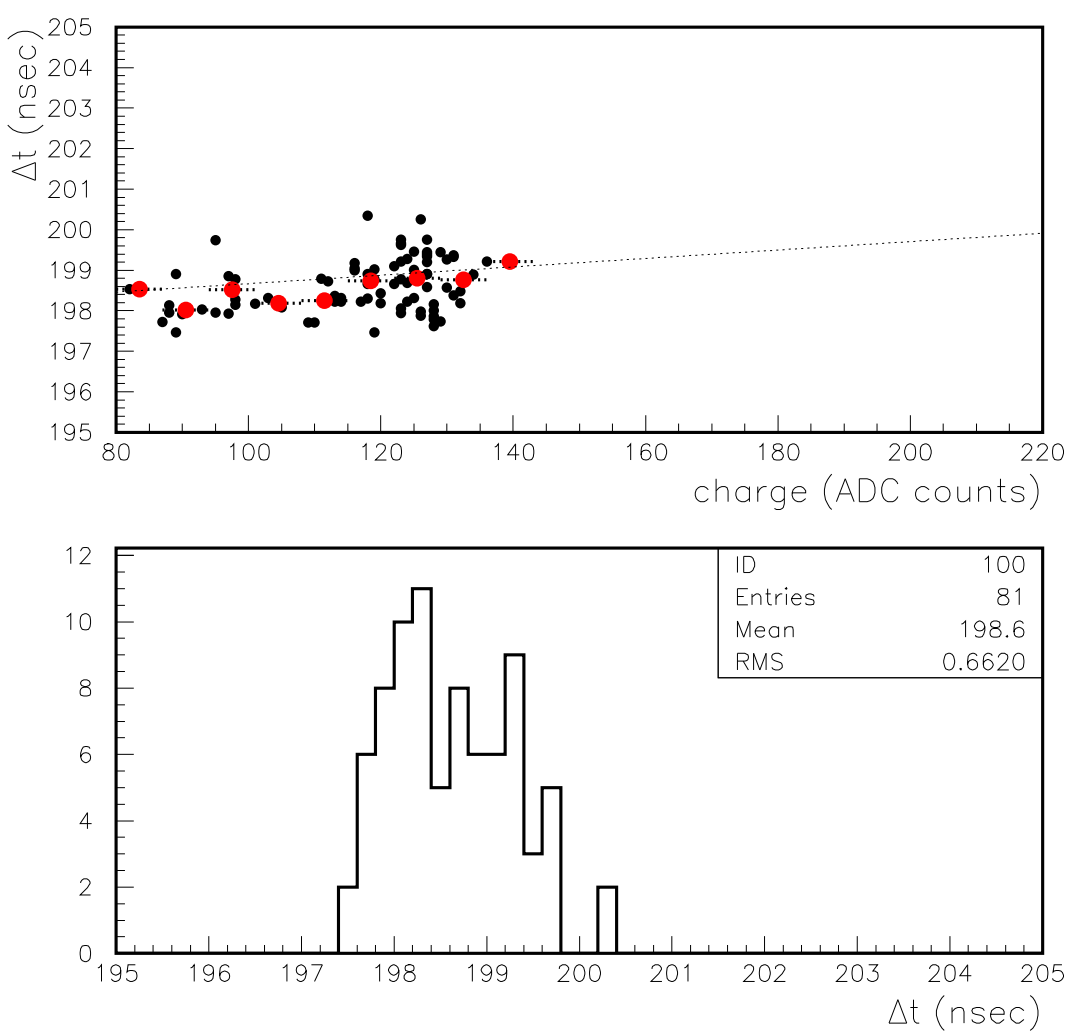

Figure 9: Upper plot: Time of discriminator firing as a function of ADC counts. The corresponding histogram of time (lower plot) has an rms noise of $0.66 \mathrm{~ns}$. The injected charge is $30 \mathrm{fC}$ at random times within a $30 \mathrm{~ns}$ acquisition window. 


\begin{tabular}{|c|c|c|}
\hline Luminosity & no ADC cut & ADC $>20$ \\
\hline $14 \cdot 10^{30} \mathrm{~cm}^{-2} \mathrm{~s}^{-1}$ & 0.102 & 0.084 \\
$36 \cdot 10^{30} \mathrm{~cm}^{-2} \mathrm{~s}^{-1}$ & 0.150 & 0.122 \\
\hline
\end{tabular}

Table 2: Average occupancy of the inner layer of the CFT as a function of luminosity (from Figure 10).

\begin{tabular}{|c|c|c|c|}
\hline Date & Luminosity & $\epsilon$ no ADC cut & $\epsilon$ ADC $>20$ \\
\hline 15 June 2003 & $68 \cdot 10^{30} \mathrm{~cm}^{-2} \mathrm{~s}^{-1}$ & 0.22 & 0.18 \\
14 December 2004 & $90.5 \cdot 10^{30} \mathrm{~cm}^{-2} \mathrm{~s}^{-1}$ & 0.27 & 0.22 \\
22 February 2005 & $136.7 \cdot 10^{30} \mathrm{~cm}^{-2} \mathrm{~s}^{-1}$ & 0.37 & 0.30 \\
13 September 2005 & $218 \cdot 10^{30} \mathrm{~cm}^{-2} \mathrm{~s}^{-1}$ & 0.55 & 0.44 \\
16 January 2007 & $294 \cdot 10^{30} \mathrm{~cm}^{-2} \mathrm{~s}^{-1}$ & 0.71 & 0.57 \\
\hline
\end{tabular}

Table 3: Design projection of the initial luminosity[8] and corresponding mean occupancy of the CFT inner layer $\epsilon$ (without and with the ADC $>20$ cut) as a function of time.

0.003 at present. However the observed occupancy of the SMT ranges from 0.005 to 0.06 depending on the SVX (is it dominated by thermal silicon and electronic noise?). For our estimate we take $n_{S i}=4$, and $\epsilon_{S i}=0.06$ independent of luminosity. Then the CFT+SMT meets bench-mark tracking performance up to a CFT occupancy $\epsilon \approx 0.29$.

Now consider the addition of the time-of-flight measurement in the CFT. If this measurement effectively divides each fiber into $N$ segments, then Equation (1) multiplied by the factor (2) becomes multiplied approximately by

$$
\times N^{2-n_{A}-n_{B}} .
$$

For our bench mark calculation we take $N \approx 2$. Then the SMT+CFT+timeof-flight can meet bench-mark tracking performance up to a CFT occupancy $\epsilon \approx 0.52$.

In summary, using the occupancies in Table 3 without the ADC cut, we estimate that the stand-alone CFT meets benchmark tracking performance up to a luminosity $\approx 55 \cdot 10^{30} \mathrm{~cm}^{-2} \mathrm{~s}^{-1}$, the SMT $+\mathrm{CFT}$ up to $\approx 100 \cdot 10^{30} \mathrm{~cm}^{-2} \mathrm{~s}^{-1}$, and the SMT+CFT+time-of-flight up to $\approx 200 \cdot 10^{30} \mathrm{~cm}^{-2} \mathrm{~s}^{-1}$. Of course more detailed studies are necessary. 


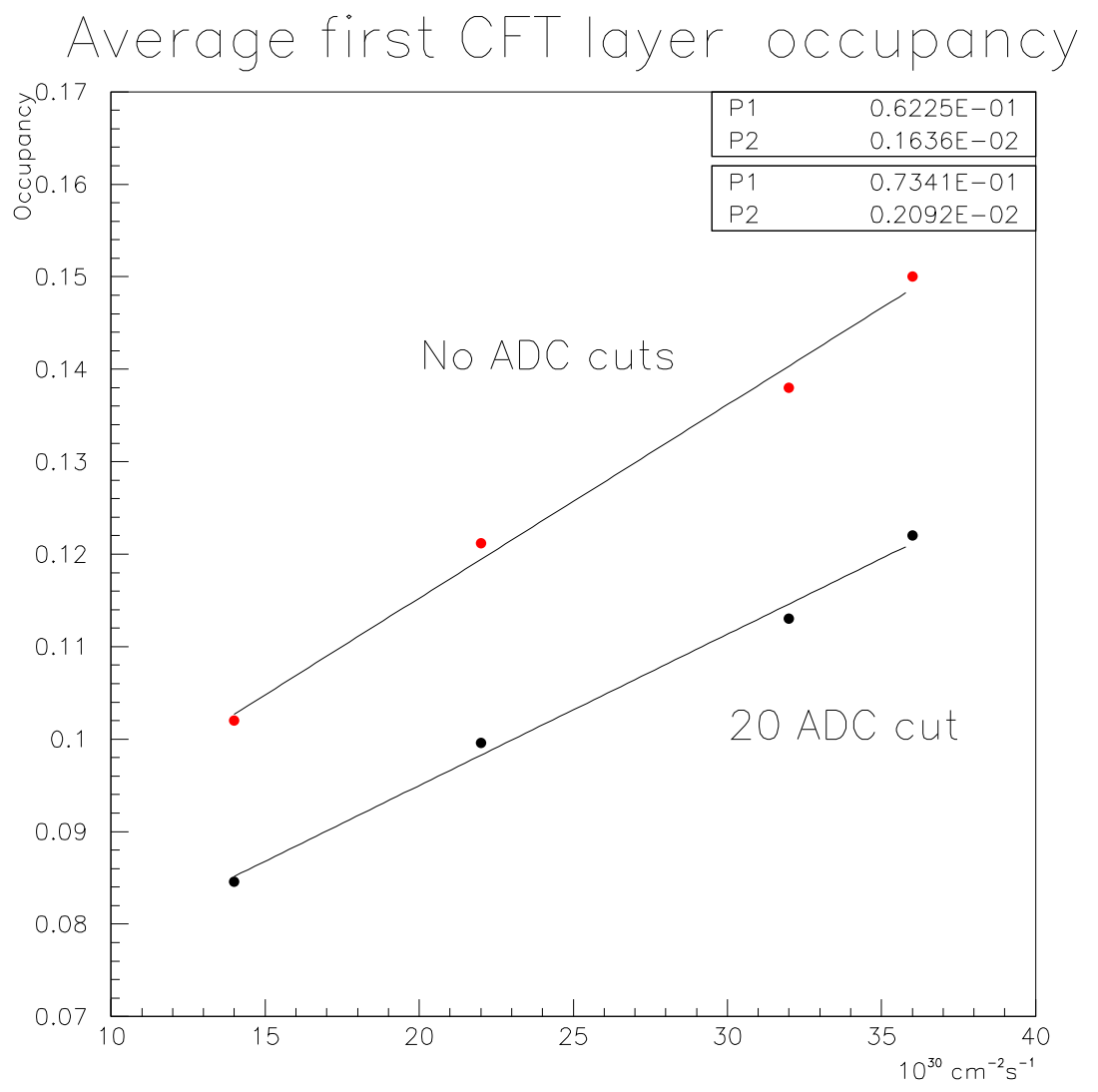

Figure 10: Measured average occupancy of the inner layer of the CFT as a function of luminosity.[7] 


\section{Conclusions}

The TriP chip and MCM II were developed to be able to operate the Central Fiber Tracker and Preshower detectors at 132ns bunch crossing time. The first submission of the TriP chip and the MCM II met all specifications. With one more submission of the TriP chip we can add a time-offlight measurement in the CFT at 396ns bunch-crossing. We have measured an $\mathrm{rms}$ resolution of $46 \mathrm{~cm}$ in the center of the scintillating fiber using the TriP chip and MCM IIb (for cosmic muons producing 8 or more photoelectrons). This resolution is dominated by the statistics of the photon arrival time and scales with the number $n$ of photo-electrons as $\propto \sqrt{n}$. [3] The resolution for hits on the near or far ends of the fibers is calculated to be of order $27 \mathrm{~cm}$ because of the higher light yields of tracks with oblique incidence, see Table 9 of [3]. The time-of-flight measurement is equivalent to approximately doubling the number of channels of the CFT. We estimate that the SMT+CFT will meet bench-mark tracking performance up to $\approx 100 \cdot 10^{30} \mathrm{~cm}^{-2} \mathrm{~s}^{-1}\left(\approx 200 \cdot 10^{30} \mathrm{~cm}^{-2} \mathrm{~s}^{-1}\right)$ without (with) the time-of-flight measurement.

\section{References}

[1] Paul Rubinov and Bruce Hoeneisen, D $\emptyset$ note 3897 (2001)

[2] P. Rubinov and B. Hoeneisen, D $\emptyset$ note 3898 (2001)

[3] J. Estrada, C. García, B. Hoeneisen, P. Rubinov, DøNote 4009(2002), also FERMILAB-TM-2226

[4] Juan Estrada, Charly García, Bruce Hoeneisen, Paul Rubinov, D $\emptyset$ Note 4076(2002), also FERMILAB-TM-2227

[5] Bruce Hoeneisen and Don Lincoln, D $\emptyset$ note 3889 (2001)

[6] Bruce Hoeneisen, D $\emptyset$ note 3842 (2001)

[7] http://d0server1.fnal.gov/projects/SciFi/ftg_meeting_minutes/index.html $\rightarrow$ Apr. 15, 2003 Meeting $\rightarrow$ Average first CFT layer occupancy.

[8] "The Run II Luminosity Upgrade at the Fermilab Tevatron. Project Plan and Resource-Loaded Schedule" June 15, 2003. 